REVISTA PROYECCIONES: 151-177.

JORNADAS MATEMATICAS, AGOSTO 1983:

\title{
¿QUÉ ES GEOESTADÍSTICA?
}

PATRICIO ROJAS MORERA*

Los Geólogos e Ingenieros de Minas, tienen una idea pragmátị ca de la noción de "variable regionalizada", en general cualquier característica geológica cuyo valor numérico esté relacionado con su po sición espacial se puede considerar como una variable regionalizada.

Conocido el problema de sobre y subestimación de reservas mi nerales en un yacimiento y también conocidas las limitaciones de los métodos estádisticos clásicos (sobre todo por no considerar la posición espacial de las muestras en que se trabaja). Los profesionales dedicados al cálculo de reservas de minerales, iniciaron la búsqueda de métodos que solucionaran dos grandes problemas

* Master en Estadística Matemática.

Profesor Departamento Matematicas, Facultad de Ciencias. Universidad del Norte. 
(i) La sobre y subestimación

(ii) La incertidumbre frente a los resultados obtenidos, al no conocer los errores de estimación.

Es así que surgen como grandes soluciones para el problema planteado en (i), el método de polígonos, el método del inverso del cuadrado de la distancia, pero continuaba sin solución el problema planteado en (ii).

G. Matheron, en el año 1962, introduce la noción de variable regionalizada cuya concepción teórica nace de la necesidad de diferen ciar datos numéricos, aparentemente del mismo tipo, pero que su posición espacial, los hace distintos para análisis geológicos.

Analicemos este concepto con un ejemplo. Consideremos tres conjuntos de datos
(1) $\begin{array}{llllllllll}20 & 22 & 26 & 28 & 32 & 25 & 24 & 21 & 20 & 12\end{array}$
(2) $\begin{array}{llllllllll}20 & 32 & 28 & 26 & 12 & 25 & 20 & 21 & 24 & 22\end{array}$
(3) $\begin{array}{llllllllll}80 & 82 & 86 & 88 & 92 & 85 & 84 & 81 & 80 & 72\end{array}$

con un análisis estadístico clásico podemos ver que

$$
\begin{array}{ll}
\bar{x}_{1}=23 & s_{1}=5.42 \\
\bar{x}_{2}=23 & s_{2}=5.42 \\
\bar{x}_{3}=83 & s_{3}=5.42
\end{array}
$$

Los dos primeros conjuntos no presentan ninguna diferencia en media ni en desviación típica, el tercer conjunto se diferencia de los anteriores en el valor medio, pero mantiene la misma desviación típica.

Si diseñamos un nuevo parámetro que podríamos llamar promedio de las diferencias cuadráticas, definido por la fórmula

$$
\mathrm{PDC}=\frac{1}{\mathrm{~N}-1} \sum_{i=1}^{\mathrm{N}-1}\left(\mathrm{z}_{i}-\mathrm{z}_{i+1}\right)^{2}
$$


donde $\mathrm{N}$ es el número de datos e $\mathrm{i}$ es el subíndice de posición de los datos, obtenemos

$$
\begin{aligned}
& \mathrm{PDC}_{1}=17.6 \\
& \mathrm{PDC}_{2}=48.8 \\
& \mathrm{PDC}_{3}=17.6
\end{aligned}
$$

Estas 3 últimas cantidades las podemos interpretar de la si guiente forma, el primer y el tercer conjunto, a pesar de tener diferentes medias, son igualmente "regulares", en cuanto a las discrepan cias de valores vecinos, en cambio, el primer y segundo conjunto, a pesar de tener la misma media, son bastantes diferentes en cuanto a su regularidad. De modo que este parámetro (PDC) nos permite discriminar respecto de la "regularidad espacial" que presentan los datos.

Usando el parámetro PDC, podemos definir una función llamada semivariograma, de la siguiente forma

$$
\gamma(h)=\frac{1}{2(N-h)} \sum_{i=1}^{N-h}\left(z_{i}-z_{i+h}\right)^{2}
$$

donde $h$ puede tomar valores enteros, el valor de $\gamma(h)$ para distintos valores de h nos da la discrepancia entre muestras separadas a distancias factores enteros de $h$.

Obteniendo $\gamma(h)$ para varios valores de $h$, es posible graficar $\gamma(h)$ en función de $h$, al gráfico resultante, llamado semivariograma experimental, se le puede ajustar un modelo teórico.

El semivariograma permite entre otras cosas determinar aniso tropías del yacimiento (calculando el semivariograma en distintas direcciones), zonas de influencia (conocido como alcance y que represen ta la distancia en la cual influye el valor de una muestra sobre las muestras vecinas), correlaciones espaciales (conocida como meseta y representada en el gráfico por la altura máxima que alcanza).

La teoría nos indica que para diferentes yacimientos los semivariogramas teóricos más usuales son los siguientes 
$\gamma(\mathrm{h})=\mathrm{p}|\mathrm{h}|$

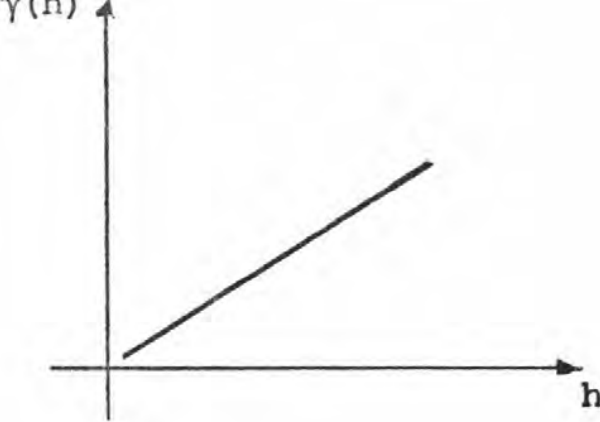

SEMIVARIOGRAMA DE DE WIJS

$\gamma(h)=3 \alpha \log \frac{h}{l}$

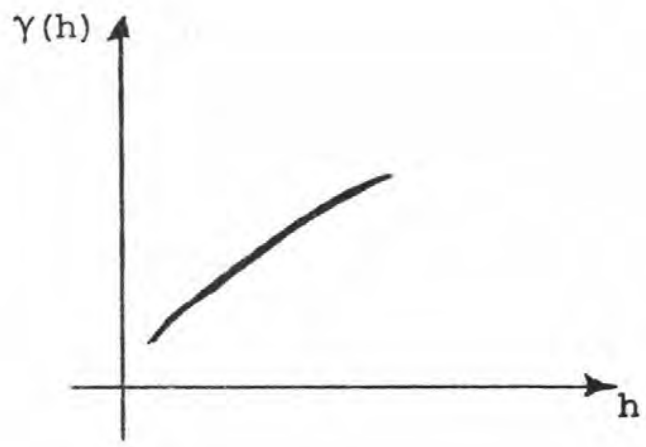

SEMIVARIOGRAMA ESFERICO DE MATHERON $\gamma(h)= \begin{cases}c\left(\frac{3}{2} \frac{h}{a}-\frac{1}{2} \frac{h^{3}}{a^{3}}\right) & h \leqslant a \\ c & h>a\end{cases}$

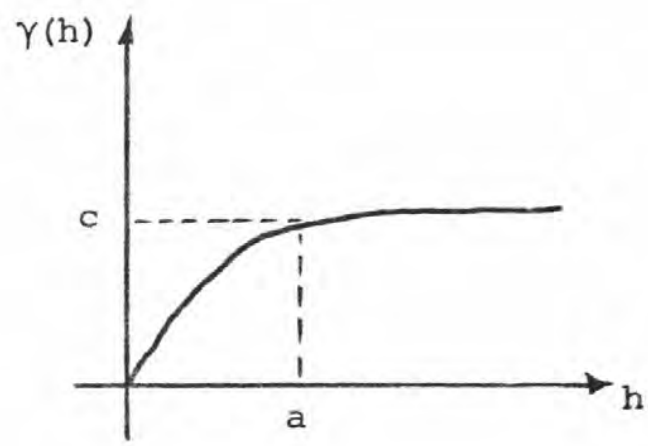

SEMIVARIOGRAMA EXPONENCIAL

$\gamma(h)=c\left(1-e^{-\frac{h}{a}}\right)$

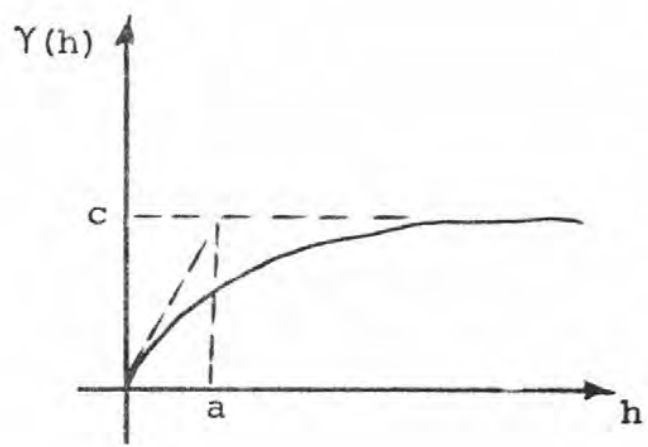

SEMIVARIOGRAMA DE GAUSS

$\gamma(h)=c\left(1-e^{-\frac{h^{2}}{a^{2}}}\right)$

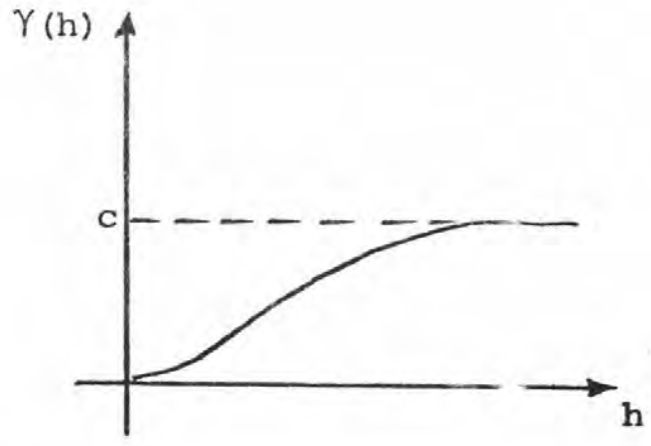


Se nos plantea ahora el problema de estimación global de un yacimiento, para lo cual es necesario manejar dos conceptos, la varianza de estimación y la varianza de extensión, que nos permitirán encontrar intervalos de confianza para nuestras estimaciones, el con cepto de varianza de estimación es simple y corresponde al promedio de las varianzas de extensión de las muestras disponibles, es decir si $\sigma_{E S}^{2}$ es la varianza estimación, $\sigma_{E X}^{2}$ es la varianza de extensión, en tonces

$$
\sigma_{E S}^{2}=\frac{1}{N} \sigma_{E X}^{2}
$$

El concepto de varianza de extensión es matemáticamente mucho más complicado y se refiere teóricamente al error cometido al estimar, por ejemplo, un panel por su sondeo central, o al estimar un bloque por sondeos en sus vértices. Esta varianza depende en general del semivariograma y de la configuración del elemento que se desea es timar. El cálculo directo de estas varianzas, se debe hacer a través de complicadas fórmulas matemáticas como la que mostramos a continuación

$$
\begin{gathered}
\sigma_{E X}^{2}=\frac{2}{v v^{\prime}} \int_{v} \int_{v^{\prime}} \gamma(x-y) \cdot d x d y-\frac{1}{v^{2}} \int_{v} \int_{v} \gamma(x-y) d x d y- \\
-\frac{1}{v^{\prime 2}} \int_{v^{\prime}} \int_{v^{\prime}} \gamma(x-y) d x d y
\end{gathered}
$$

Pero la solución aproximada de estas integrales se encuentran disponibles en ábacos como el que se muestra a continuación. 


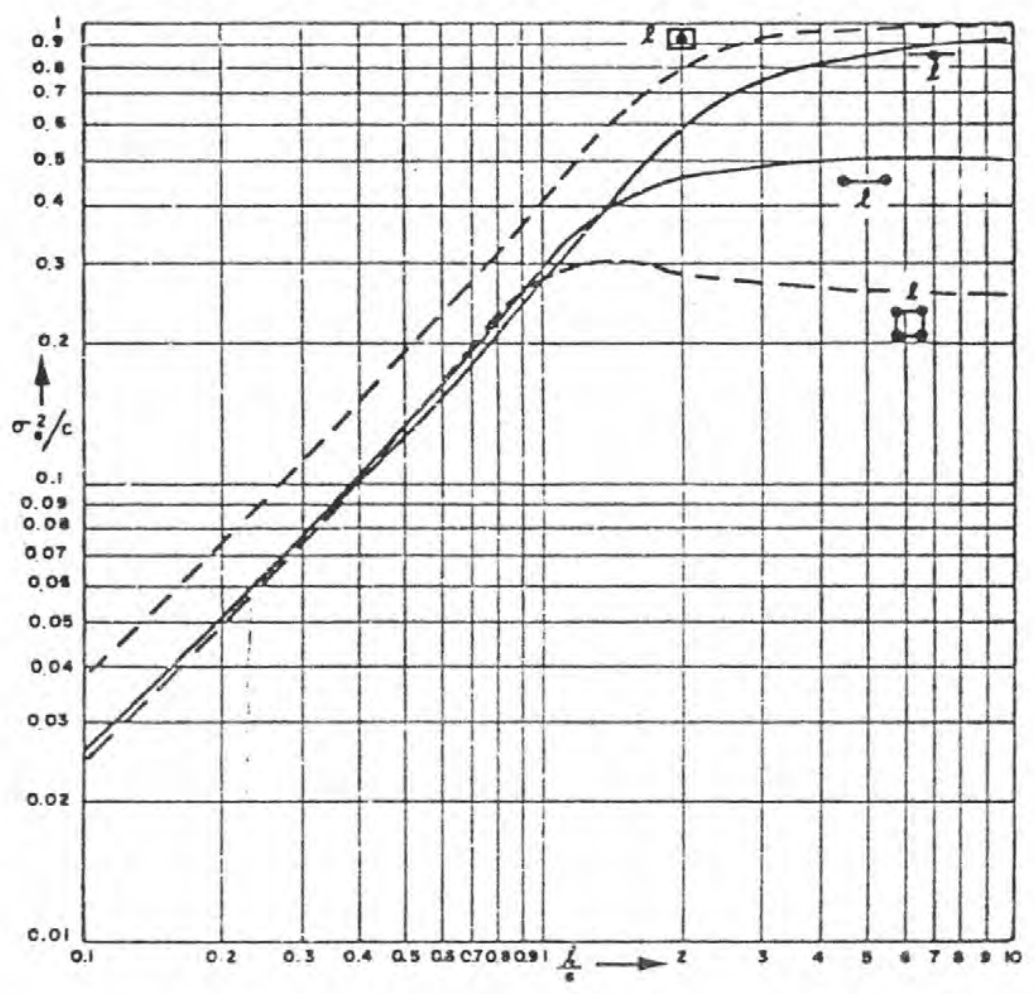

FIGURA B:

Varianzas de extensión para el esquema de Matheron (válido para semi variogramas isótropos) y para las configuraciones indicadas.

Conocidas las varianzas de estimación podemos diseñar inter valos de confianza como los siguientes

$m=m_{0}^{*} \pm \sigma_{E S} \quad$ de $68 \%$ de probabilidad

$m=m_{0}^{*} \pm 2 \sigma_{E S}$ de $95 \%$ de probabilidad

$m=m_{0}^{*} \pm 3 \sigma_{E S}$ de $99 \%$ de probabilidad 
Donde $m_{0}^{*}=\frac{\sum_{i=1}^{N} z_{i}}{N}$

Veamos ahora el problema de estimación local, aquí se trata de estimar la ley de un panel en base a la información disponible, aquí aparecen como solución los métodos geométricos mencionados anteriormente (polígonos, inverso del cuadrado de la distancia, etc.), pe ro la teoría nos indica que el mejor estimador local es el estimador del Krigeage, que consiste en un estimador lineal que atribuye pesos a la ley de cada muestra; estos pesos se calculan de manera de hacer mínima la varianza de estimación, considerando las características geométricas (formas, dimensiones y ubicación relativas de las mues tras en el panel). Teóricamente se trata de encontrar un estimador que presente dos características esenciales

(i) Es insesgado, es decir si $z$ es la verdadera ley del panel y $\mathrm{z}_{\mathrm{k}}^{*}$ es el estimador de Krigeage, se tiene que

$$
E\left(Z-Z_{k}^{*}\right)=0
$$

(ii) Es óptimo, es decir la varianza de estimación $E\left(z-z_{k}^{\star}\right)^{2}$ es mínima.

Aplicando estas condiciones y el formalismo de Lagrange, para la minimización de la función se obtiene el sistema de ecuaciones

$$
\left\{\begin{array}{l}
\sum_{j=1}^{N} \lambda_{j} \gamma_{i j}=\gamma_{i v}-\mu \quad i=1,2, \ldots N \\
\sum_{j=1}^{N} \lambda_{j}=1
\end{array}\right.
$$

cuya solución nos permite encontrar los valores de $\lambda_{i}$ ( $i=1,2, \ldots$ N), de modo que podemos calcular el estimador de Krigeage como

$$
z_{k}^{*}=\sum_{i=1}^{N} \lambda_{i} z_{i}
$$


La varianza de estimación es

$$
\sigma_{k}^{2}=\sum_{i=1}^{N} \lambda_{i} \gamma_{i v}-\gamma_{v, v}+\mu
$$

De modo que podemos construir intervalos de confianza para $\mathrm{Z}_{\mathrm{p}}$ (Ley del Panel).

$$
\begin{array}{ll}
z_{p}=z_{k}^{*} \pm \sigma_{k} & \text { de } 68 \% \text { de probabilidad } \\
z_{p}=z_{k}^{*} \pm 2 \sigma_{k} & \text { de } 95 \% \text { de probabilidad } \\
z_{p}=z_{k}^{*} \pm 3 \sigma_{k} & \text { de } 99 \% \text { de probabilidad }
\end{array}
$$

Es necesario dar una breve explicación sobre la simbología usada en el sistema de ecuaciones, mencionado anteriormente. Suponga mos que deseamos una estimación local de un panel de 200 x $200 \mathrm{mts}^{2}$, por un sondeo central, y que su semivariograma da un alcance aproxima do de $420 \mathrm{mts}$, en estas condiciones tenemos la siguiente configuración

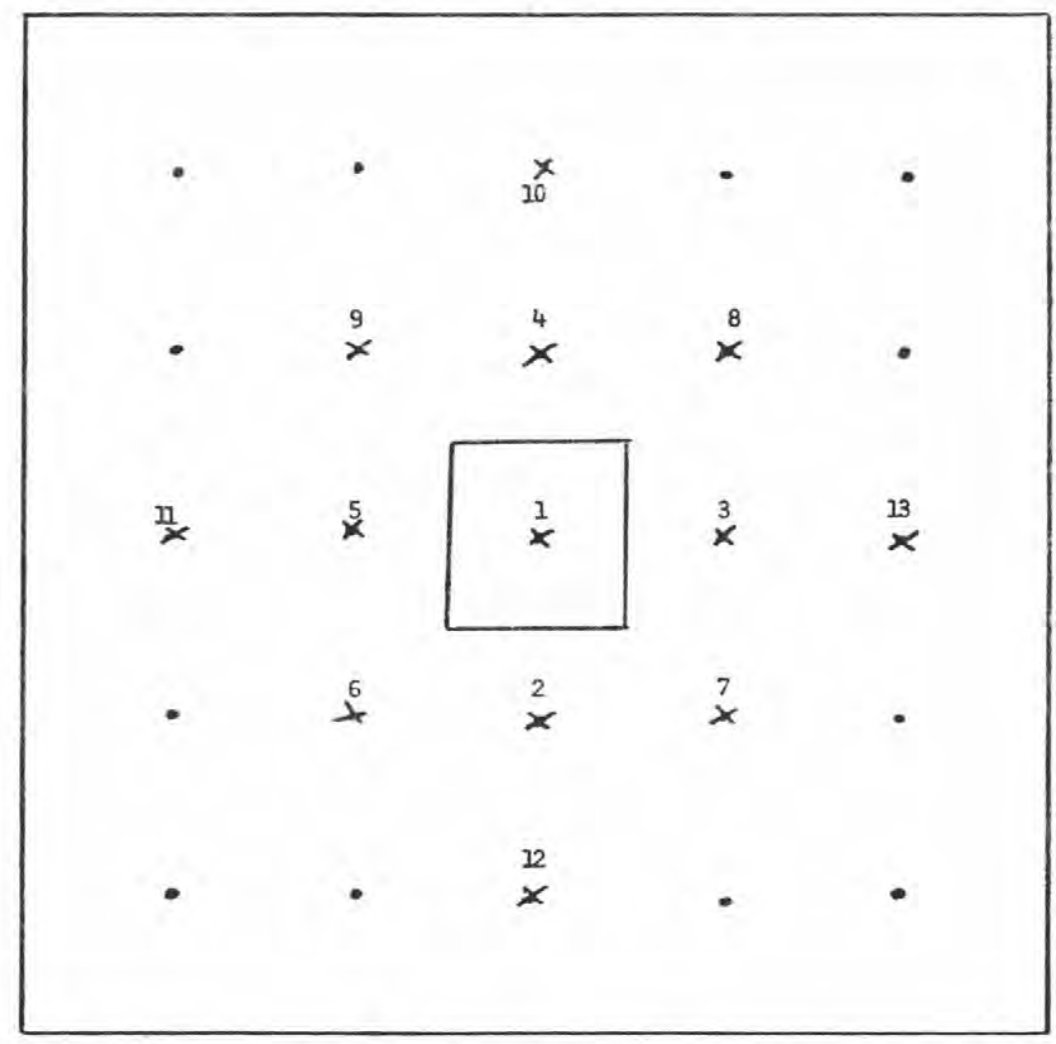


En la figura $C$ hemos trazado un círculo de radio 420 mts. y hemos marcado con cruces las muestras dentro del alcance y que usare mos para conseguir el sistema de ecuaciones.

Entonces tendríamos un sistema de 14 ecuaciones simultáneas por resolver, pero la teoría nos dice que podemos aplicar dos propie dades que permiten reducir el sistema de ecuaciones

(i) Efecto de simetría, los ponderadores a igual distancia del centro del panel son iguales, es decir podemos anotar

$$
\begin{aligned}
& \lambda_{1}=\mathrm{A} \\
& \lambda_{2}=\lambda_{3}=\lambda_{4}=\lambda_{5}=\mathrm{B} \\
& \lambda_{6}=\lambda_{7}=\lambda_{8}=\lambda_{9}=\mathrm{C} \\
& \lambda_{10}=\lambda_{11}=\lambda_{12}=\lambda_{13}=\mathrm{D}
\end{aligned}
$$

(ii) Efecto de pantalla, para los ponderadores que se encuentran en línea recta, con el centro del panel, sólo se considera el más cercano, los otros son nulos, así tenemos

$$
\lambda_{10}=\lambda_{11}=\lambda_{12}=\lambda_{13}=0
$$

De modo que aplicando los efectos de simetría y de pantalla nuestro sistema original de 14 ecuaciones nos queda de sólo 5 ecuaciones.

Al desarrollar las sumatorias aparecen términos, por ejemplo, de la forma $\gamma_{13}$ que se refiere al valor de $\gamma$ (distancia entre la muestra 1 y la 3). En la figura $c$, vemos que esta distancia es de 200 metros, luego $\gamma_{13}=\gamma(200)$ que se puede calcular directamente de la expresión matemática para el semivariograma, analogamente $\gamma_{17}=\gamma(263)$.

También aparecen en el segundo miembro de las ecuaciones términos de la forma $\gamma_{1 v}$ ' que corresponde a la varianza de extensión de la muestra 1 sobre el panel, y que se calcula en base a funciones auxi liares, que posteriormente son leídos en ábacos. En las figuras siguientes se muestran algunas fórmulas de aproximación para diferentes configuraciones y también ábacos que permiten calcular las funciones auxiliares. 
CONFIGURACION

\section{SONDEO CENTRAL}

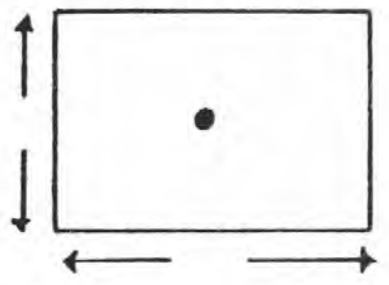

SONDEO EN UN VERTICE

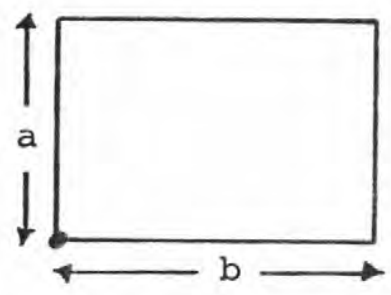

SONDEO INTERIOR

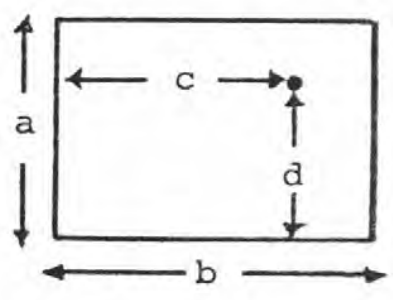

SONDEO EXTERIOR

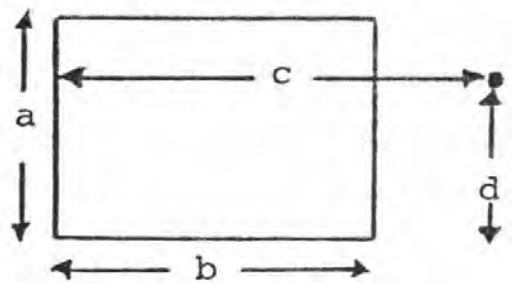

VARIANZA DE EXTENSION

$$
2 \mathrm{H}\left(\frac{\mathrm{a}}{2}, \frac{\mathrm{b}}{2}\right)-\mathrm{F}(\mathrm{a}, \mathrm{b})
$$

$2 H(a, b)-F(a, b)$

$$
\begin{aligned}
& \frac{(a-c)(b-d)}{a b} \text { H }(a-c, b-d)+\frac{c d}{a b} H(c, d)+ \\
& \frac{c(b-d)}{a b} H(c, b-d)+\frac{d(a-c)}{a b} \text { H }(a-c, d)
\end{aligned}
$$

$$
\begin{aligned}
& \frac{c(b-d)}{a b} H(c, b-d)+\frac{c d}{a b} H(c, d)- \\
& -\frac{(b-d)(c-a)}{a b} H(b-d, c-a)- \\
& -\frac{d(c-a)}{a b} \text { H }(d, c-a)
\end{aligned}
$$

FIGURA D:

Fórmulas de aproximación para calcular varianzas de extensión para diversas configuraciones. 


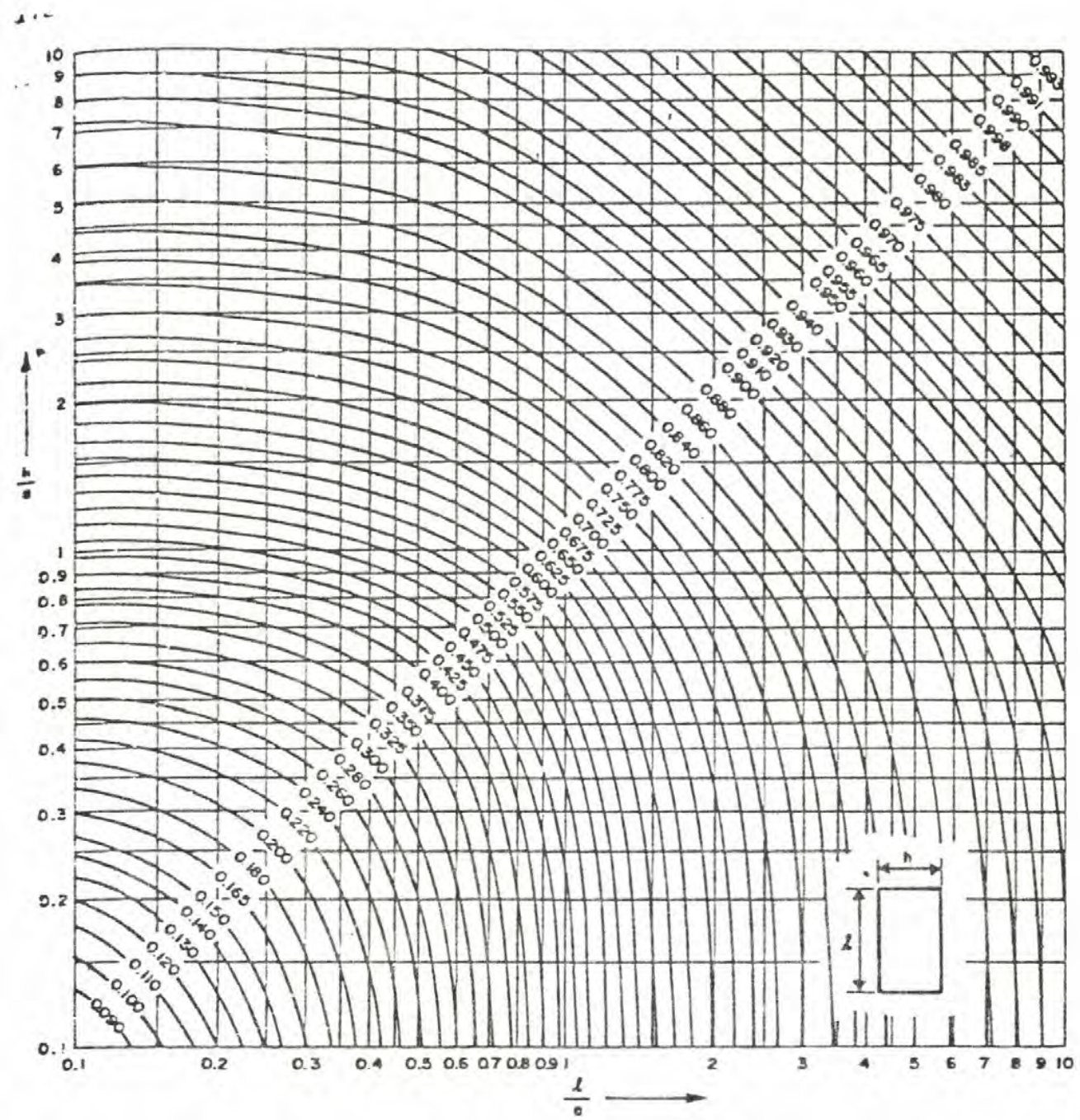

FIGURA E:

Abaco para calcular la función auxiliar $F(a, b)$ para el esquema de Matheron. 

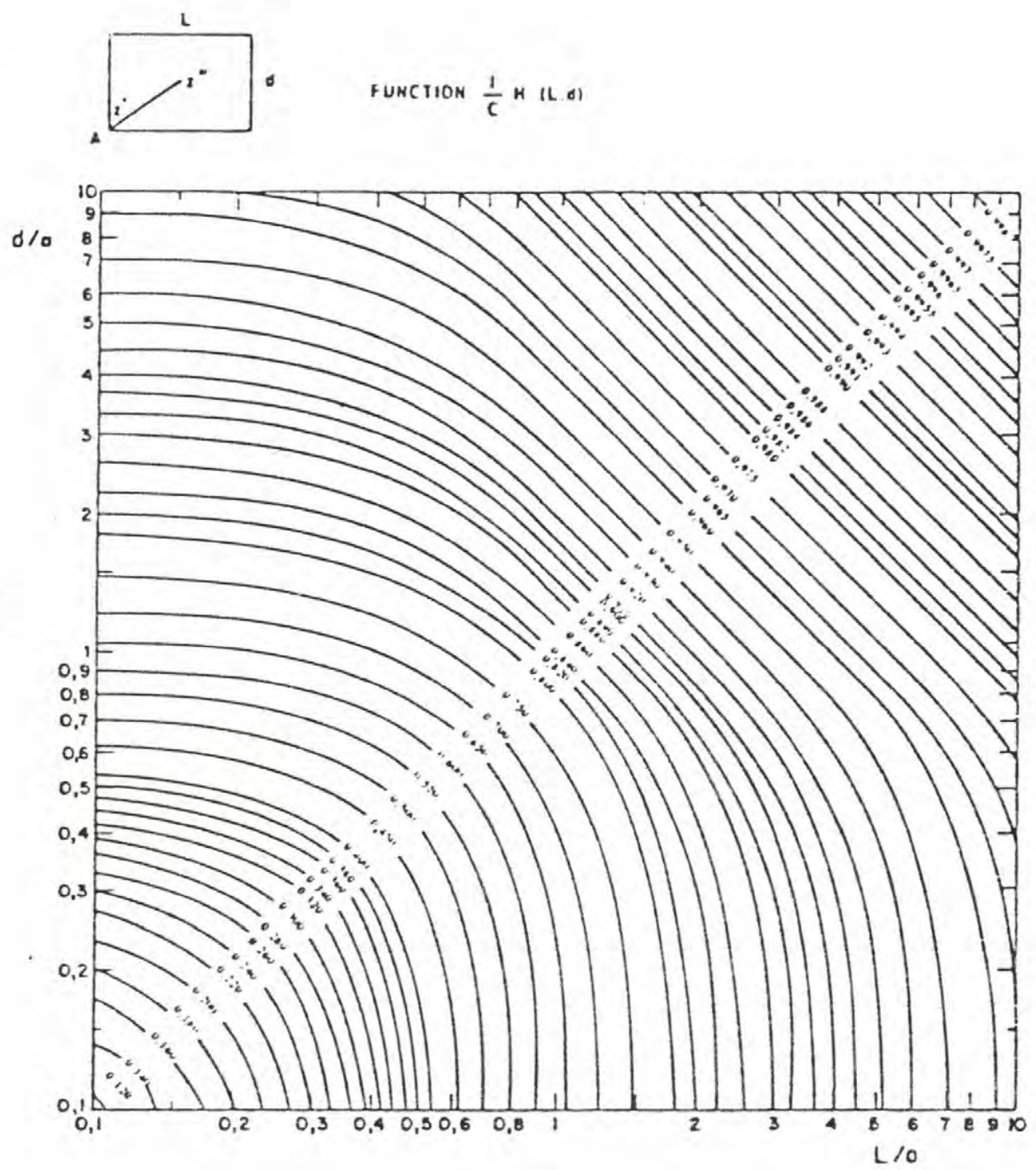

FIGURA F:

Abaco para calcular la función auxiliar $\mathrm{H}(\mathrm{a}, \mathrm{b})$ para el esquema de Matheron.

Para aclarar el uso de las fórmulas de aproximación y los ábacos desarrollaremos la ecuación correspondiente a $i=3$ para la configuración de la Figura c.

$$
\begin{gathered}
\lambda_{1} \gamma_{31}+\lambda_{2} \gamma_{32}+\lambda_{3} \gamma_{33}+\lambda_{4} \gamma_{34}+\lambda_{5} \gamma_{35}+\lambda_{6} \gamma_{36}+\lambda_{7} \gamma_{37}+\lambda_{8} \gamma_{38}+ \\
+\lambda_{9} \gamma_{39}=\gamma_{3 v}-\mu
\end{gathered}
$$




$$
\begin{aligned}
& \gamma_{31}=\gamma(200) \\
& \gamma_{34}=\gamma(283) \\
& \gamma_{37}=\gamma(200) \\
& \gamma_{32}=\gamma(283) \\
& \gamma_{35}=\gamma(400) \\
& \gamma_{38}=\gamma(200) \\
& \gamma_{33}=\gamma(0)=0 \\
& \gamma_{36}=\gamma(447) \\
& \gamma_{39}=\gamma(447) \\
& \lambda_{1}=\mathrm{A} \\
& \lambda_{2}=\lambda_{3}=\lambda_{4}=\lambda_{5}=\mathrm{B} \\
& \lambda_{6}=\lambda_{7}=\lambda_{8}=\lambda_{9}=\mathrm{c}
\end{aligned}
$$

tenemos

$$
[\gamma(200)] A+[2 \gamma(283)+\gamma(400)] B+2[\gamma(447)+\gamma(200)] C=\gamma_{3 V}-\mu
$$

Donde los valores de $\gamma$ se pueden calcular directamente de la expresión matemática del semivariograma.

Para calcular $\gamma_{3 v}$, usamos el caso 4 de la Figura $D$ en la cual hacemos $a=200, b=200, c=300, d=100$. Luego nos queda

$$
\gamma_{3 V}=\frac{3}{2} H(300,100)-\frac{1}{2} H(100,100)
$$

Estos valores de H pueden ser leídos de la Figura F de esta forma comple tamos la ecuación para $i=3$.

A continuación veamos un caso práctico en un yacimiento simulado.

Problema: Supongamos que tenemos un yacimiento de $2200 \times 1800 \mathrm{mts}^{2}$. Se desea hacer un reconocimiento secuencial, haciendo estimaciones globales y locales.

Implantamos en nuestro yacimiento una malla de $400 \times 400 \mathrm{mts}^{2}$ y hacemos un sondeo en el cuadrante superior izquierdo de cada panel como se indica en la figura 


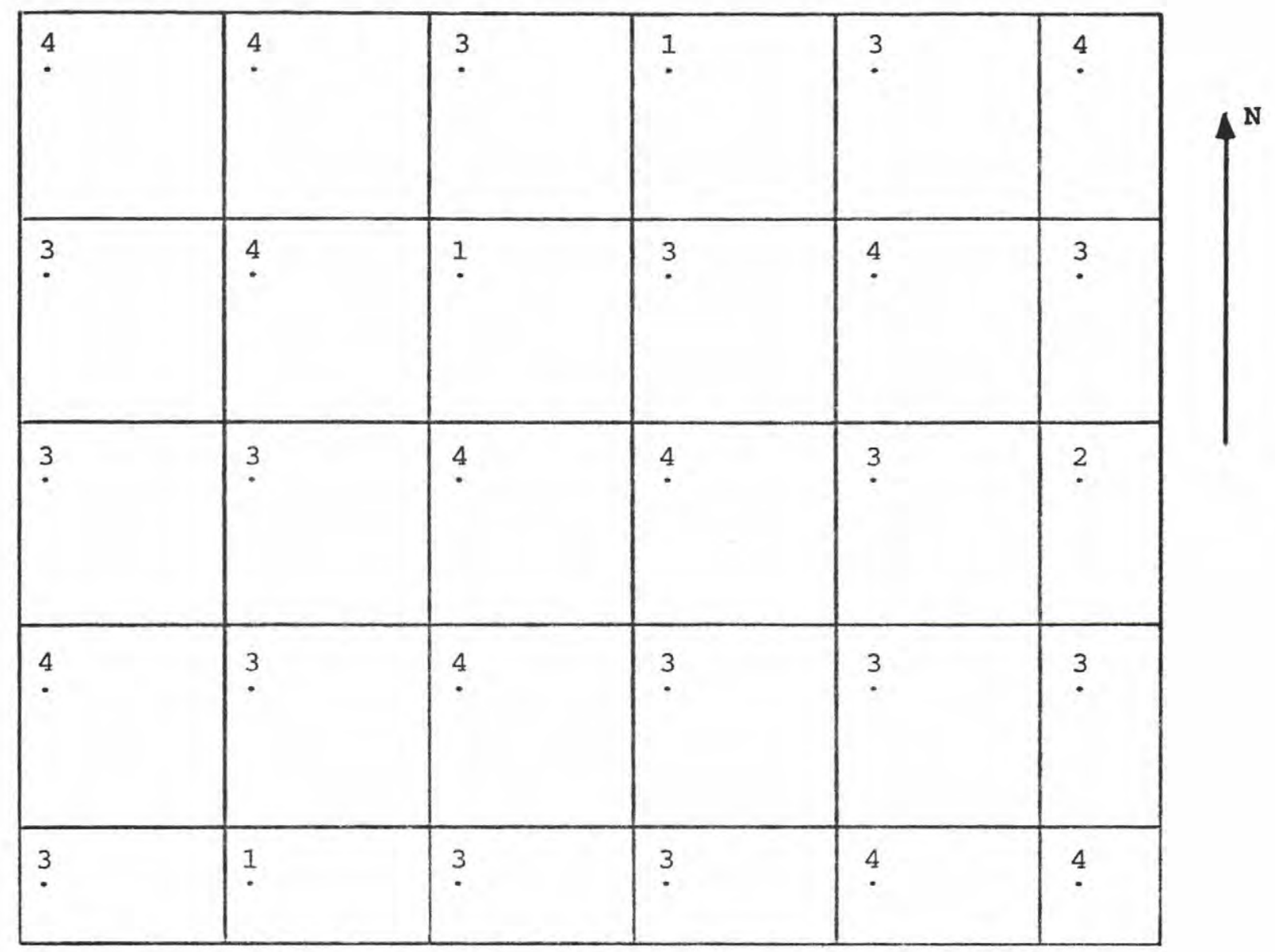

FIGURA 1:

Malla de $400 \times 400 \mathrm{mts}^{2}$ impuesta sobre el yacimiento. (Primera campaña de sondeos).

Construyendo los semivariogramas en las direcciones norte-sur y este-oeste, nos encontramos que con tan pocos datos los semivariogra mas no nos entregan información suficiente para ajustar algunos de los modelos. 


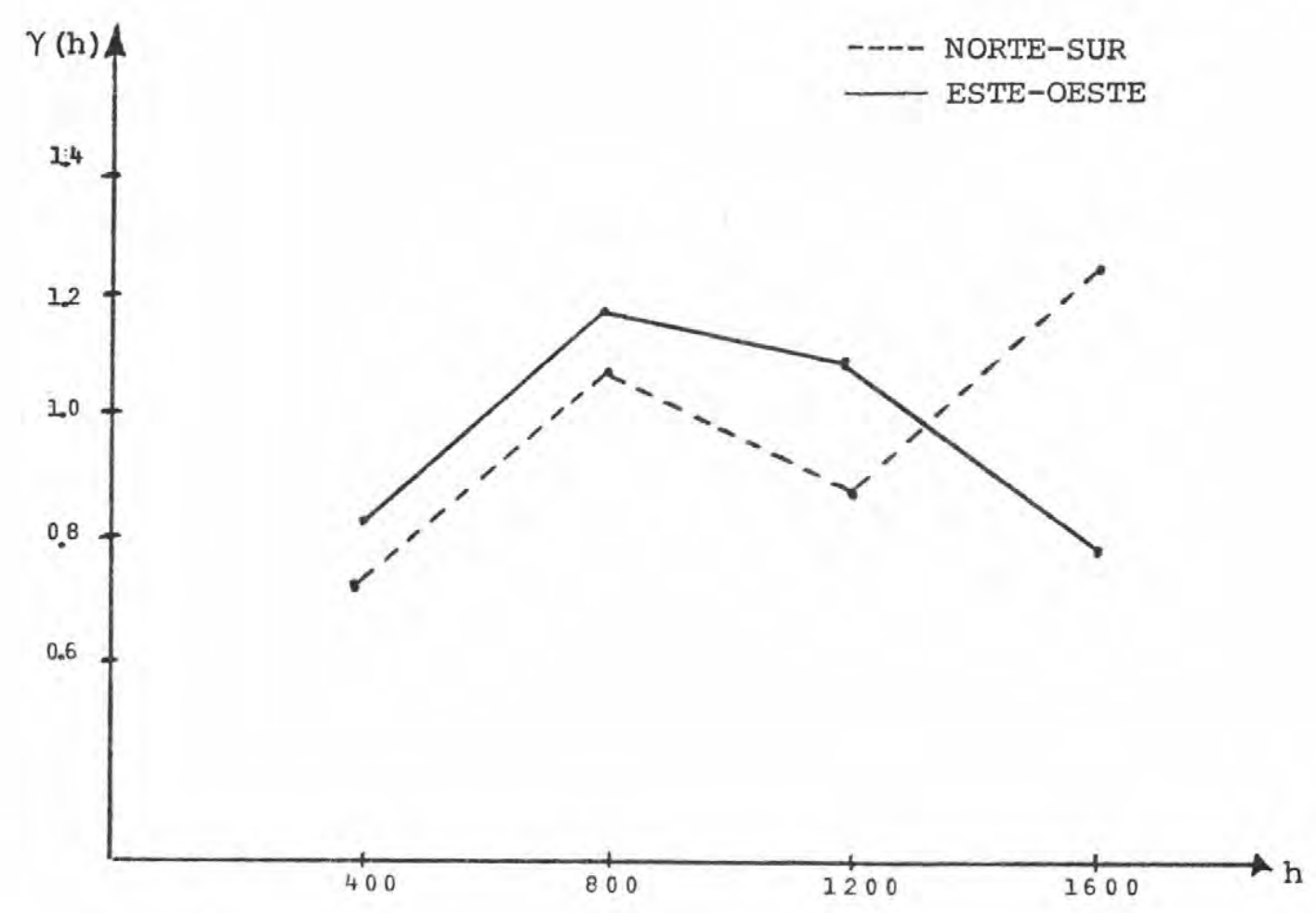

FIGURA 2:

Semivariogramas correspondientes a la primera campaña de sondeos.

Pero de todas maneras podemos obtener la siguiente información

(i) La poca variación vertical nos indica que la correlación espacial entre las muestras es despreciable.

(ii) El alcance de las muestras está entre 400 y 800 mts.

(iii) La meseta es aproximadamente 1.

También podríamos hacer una estimación global, calculando la varianza de extensión, pero para ello necesitamos conocer el semivario grama asociado al yacimiento, en reemplazo podemos hacer una estimación Estadística clásica, suponiendo que los datos ajustan una distribución normal. Obtenemos los siguientes valores

$$
\begin{aligned}
& \mathrm{m}_{0}=3.13 \\
& \mathrm{~s}=0.88 \\
& \mathrm{~m}=3.13 \pm 1.76, \text { o equivalentemente } \\
& \mathrm{m}=3.33 \pm 56 \%, \text { es decir estamos haciendo una estimación }
\end{aligned}
$$

global con un 56\% de error. 
Es necesario entonces realizar una segunda campaña de sondeos, para lo cual decidimos entre varias alternativas posibles (y pensando en que podemos usar los sondeos ya realizados) en implantar una malla de 200 x $200 \mathrm{mts}^{2}$ y hacer sondeos centrales, con 10 cual obtenemos la siguiente figura

\begin{tabular}{|l|l|l|l|l|l|l|l|l|l|l|}
\hline 4 & 1 & 4 & 3 & 3 & 2 & 1 & 2 & 3 & 4 & 4 \\
\hline 4 & 3 & 4 & 4 & 4 & 4 & 3 & 3 & 3 & 4 & 3 \\
\hline 3 & 1 & 4 & 4 & 1 & 3 & 3 & 4 & 4 & 3 & 3 \\
\hline 1 & 3 & 3 & 4 & 3 & 2 & 4 & 4 & 2 & 1 & 1 \\
\hline 3 & 4 & 3 & 2 & 4 & 1 & 4 & 4 & 3 & 1 & 2 \\
\hline 2 & 3 & 2 & 2 & 4 & 3 & 3 & 3 & 4 & 3 & 3 \\
\hline 4 & 3 & 3 & 3 & 4 & 4 & 3 & 3 & 3 & 4 & 3 \\
\hline 4 & 2 & 1 & 2 & 4 & 4 & 2 & 2 & 3 & 3 & 4 \\
\hline 3 & 1 & 1 & 3 & 3 & 3 & 3 & 3 & 4 & 2 & 4 \\
\hline
\end{tabular}

FIGURA 3;

Malla de $200 \times 200 \mathrm{mts}^{2}$ impuesta sobre el yacimiento (segunda campaña de sondeos).

Con estos nuevos datos obtenemos los semivariogramas en las direcciones norte-sur $y$ este-oeste, que podemos verlos en la figura siguien te. 


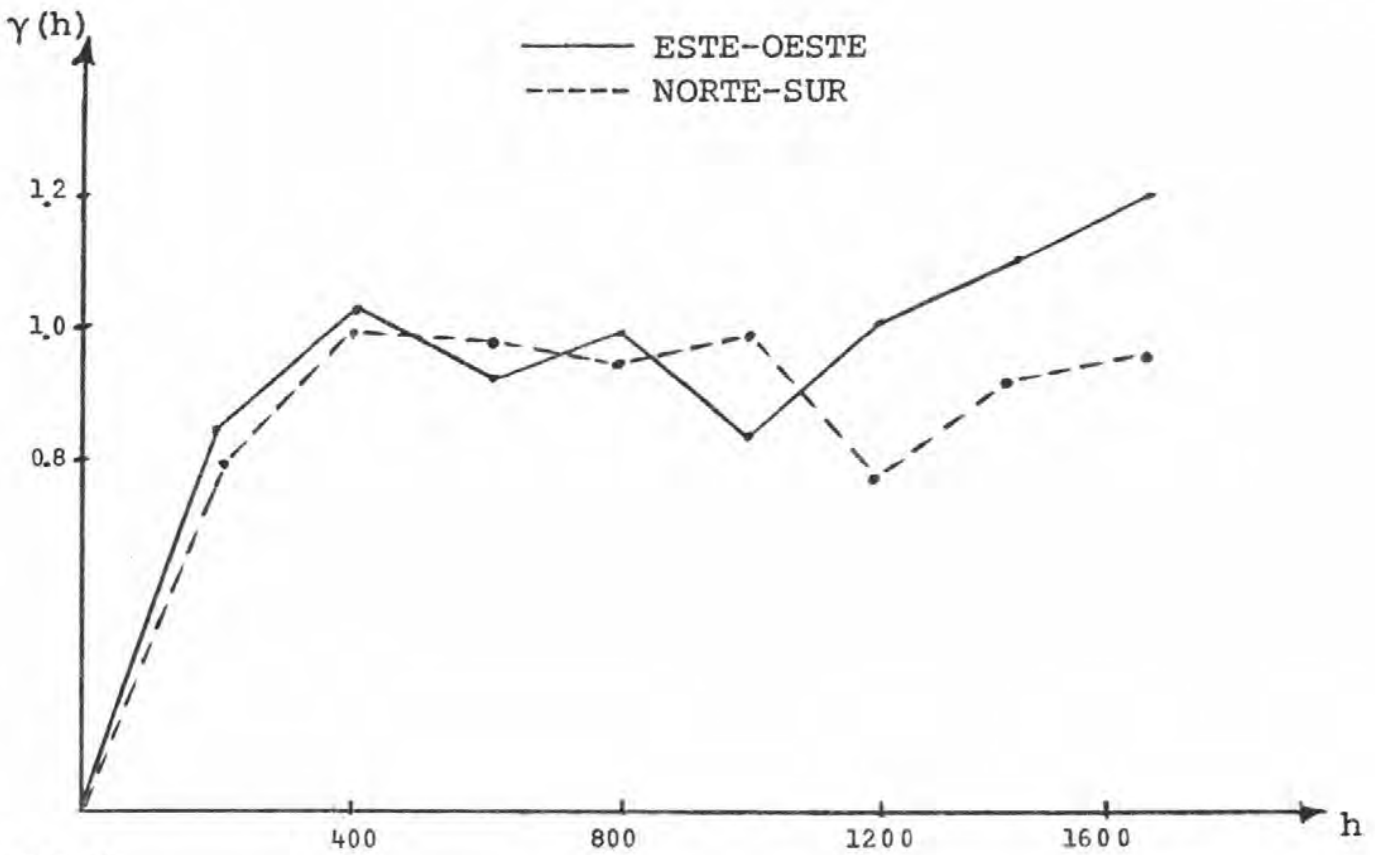

FIGURA 4:

Semivariogramas correspondientes a la segunda campaña de sondeos.

Del análisis de los semivariogramas anteriores podemos concluir que el yacimiento es isótropo, entonces podemos obtener un semi variograma promedio, que podemos ajustar a un semivariograma teórico de Matheron.

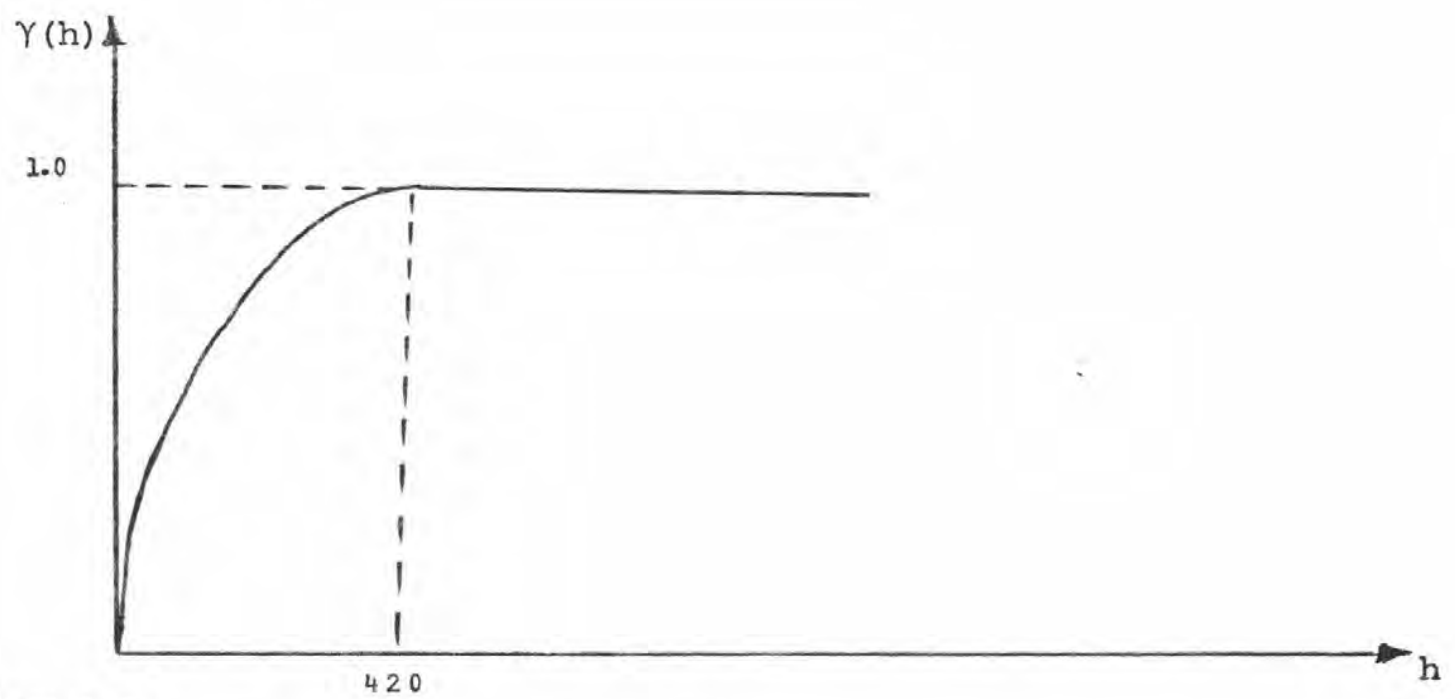

FIGURA 5:

Semivariograma teórico de Matheron. 
Del análisis del semivariograma teórico podemos concluir

i) El alcance es de 420 mts.

ii) La meseta es 1.0

Como hemos visto anteriormente, la expresión matemática general de este semivariograma de Matheron es

$$
\gamma(h)= \begin{cases}c\left(\frac{3}{2} \frac{h}{a}-\frac{1}{2} \frac{h^{3}}{a^{3}}\right) & \text { si } h<a \\ c & \text { si } h \geqslant a\end{cases}
$$

En nuestro caso particular a -420 y $c=1.0$

Luego podemos anotar

$$
\gamma(h)= \begin{cases}\frac{3}{2} \frac{h}{420}-\frac{1}{2} \frac{h^{3}}{420^{3}} & \text { si } h<420 \\ 1 & \text { si } h \geqslant 420\end{cases}
$$

Puesto que ahora conocemos el semivariograma asociado al modelo podemos hacer una estimación global para la media del yacimiento, haciendo uso de la varianza de extensión, obtenemos los siguientes valores

$$
\begin{aligned}
& \text { lores } \\
& m_{0}^{*}=\frac{\sum_{i=1}^{99} z_{i}}{99} \\
& \sigma_{E}^{2}=\frac{1}{99} \sigma_{E^{\prime}}^{2}=\frac{1}{99} \cdot 0.18=1.82 \times 10^{-3} \\
& \sigma_{E}=0.043 \\
& \therefore \quad m=m_{0}^{*} \pm 0.086
\end{aligned}
$$


Es decir al disponer de 99 sondeos tenemos un error en la es timación global de 0.086

Podríamos además calcular el error en la estimación local usando Krigeage para la siguiente configuración

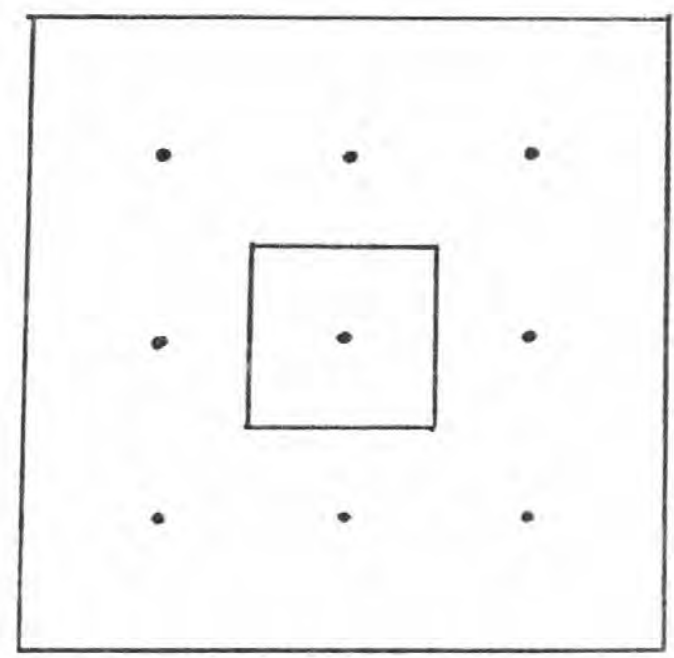

FIGURA 6:

Configuración para aplicar Krigeage a un panel con un sondeo central.

Usando la configuración anterior, el semivariograma y los ába cos adecuados, se obtiene el siguiente sistema de ecuaciones

$$
\begin{aligned}
2.64 B+3.44 C+\mu & =0.27 \\
0.66 A+2.72 B+3.32 C+\mu & =0.67 \\
0.86 A+3.32 B+3.00 C+\mu & =0.85 \\
A+4 B+4 C & =1
\end{aligned}
$$

cuya solución es
$\mathrm{A}=0.60$,
$\mathrm{B}=0.10$,
$\mathrm{C}=0.00$,
$\mu=0.00$ 
De lo cual obtenemos los siguientes valores

$z_{k}^{*}=0.60 z_{1}+0.10\left(z_{2}+z_{3}+z_{4}+z_{5}\right)$

$\sigma_{\mathrm{k}}^{2}=0.07$

Entonces una estimación local para la ley del panel, está dada por

$$
z_{p}=z_{k}^{*} \pm 0.53
$$

Es decir tenemos un exror de estimación local para la ley del panel de 0.53 .

Podemos pensar ahora en hacer más sondeos, por ejemplo en los vértices de los paneles, es decir tendríamos que hacer 120 nuevos sondeos, con los 99 anteriores tendríamos un total de 219 sondeos, con estos nuevos datos, podemos realizar una nueva estimación global, haciendo uso de los ábacos de varianza de extensión, obtenemos

$$
\begin{aligned}
& \mathrm{m}_{0}^{*}=\frac{\sum_{i=1}^{219} z_{i}}{219} \\
& \sigma_{E}^{2}=\frac{1}{219} \sigma_{E^{\prime}}^{2}=\frac{1}{219} \times 0.12=5.48 \times 10^{-4} \\
& \sigma_{E}=0.023 \\
& \therefore \quad m=m_{0} \pm 0.047
\end{aligned}
$$

Es decir al disponer de 219 sondepos tenemos un error en la esti mación global de 0.047

Usando los 219 sondeos hagamos una estimación local para la ley de un panel, mediante Krigeage. Debido al alcance del semivariograma y a la distribución de los sondeos, debemos usar la siguiente configu ración 


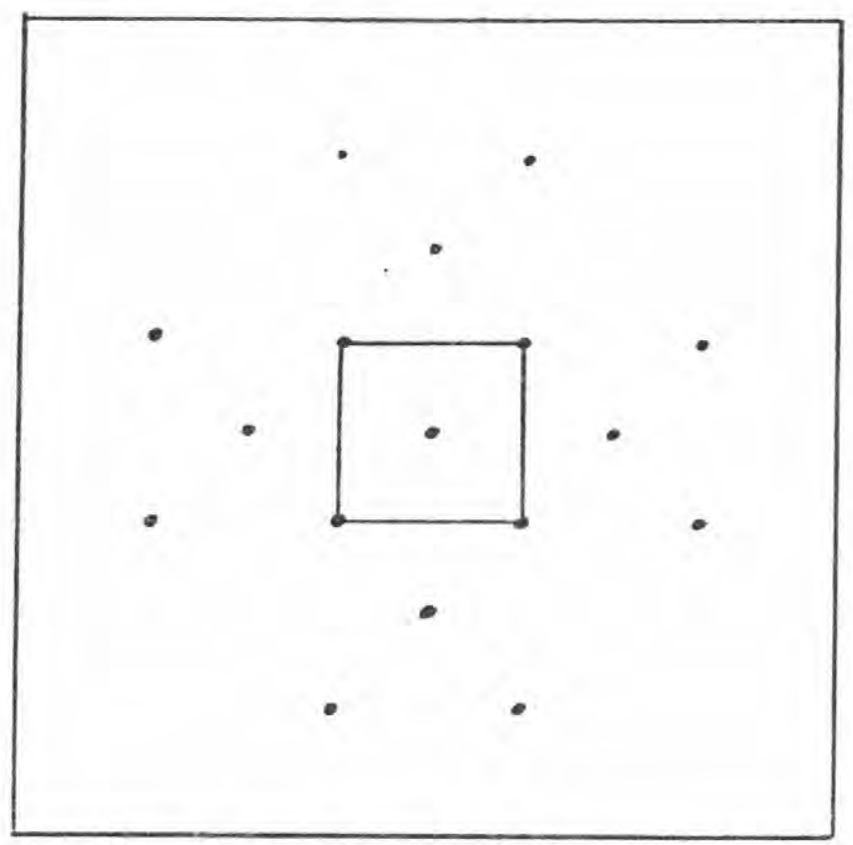

FIGURA 7:

Configuración para aplicar Kirgeage a un panel con un sondeo central y un sondeo en cada vértice.

La solución del sistema de ecuaciones conduce a la siguiente estimación para el panel

$$
z_{p}=z_{k}^{*} \pm 0.48
$$

Es decir tenemos un error de estimación local para la ley del panel de 0.48 , con $10 \mathrm{cual}$ hemos reducido nuestro error de estima ción local en un $9.4 \%$ con respecto al estudio efectuado con 99 sondeos.

Como alternativa podríamos pensar en subdividir cada panel de $200 \times 200 \mathrm{mts}^{2}$ en 4 paneles de $100 \times 100 \mathrm{mts}^{2}, \mathrm{y}$ hacer un sondeo central en cada panel de $100 \times 100 \mathrm{mts}^{2}$, para esto tendríamos que hacer 396 nuevos sondeos, sumados a los 99 ya efectuados tenemos un total de 495 sondeos. 
Una nueva estimación global nos da lo siguiente

$m_{0}^{*}=\frac{\sum_{i=1}^{495} z_{i} .}{495}$

$\sigma_{E}^{2}=\frac{1}{495} \quad \sigma_{E^{\prime}}^{2}=\frac{1}{495} \times 0.058=1.17 \times 10^{-4}$

$\sigma_{E}=0.011$

$\therefore \quad \mathrm{m}=\mathrm{m}_{0}^{*} \pm 0.022$

Es decir al disponer de 495 sondeos, tenemos un error en la es timación global de 0.022 , con lo cual hemos reducido el error de estima ción global en un $74,4 \%$ respecto a la estimación con 99 sondeos y en un $53,2 \%$, respecto a la estimación con 219 sondeos.

Usando los 495 sondeos podemos hacer una estimación local para la ley de un panel, debido al alcance del semivariograma y a la distribución de los sondeos, debemos usar la siguiente configuración

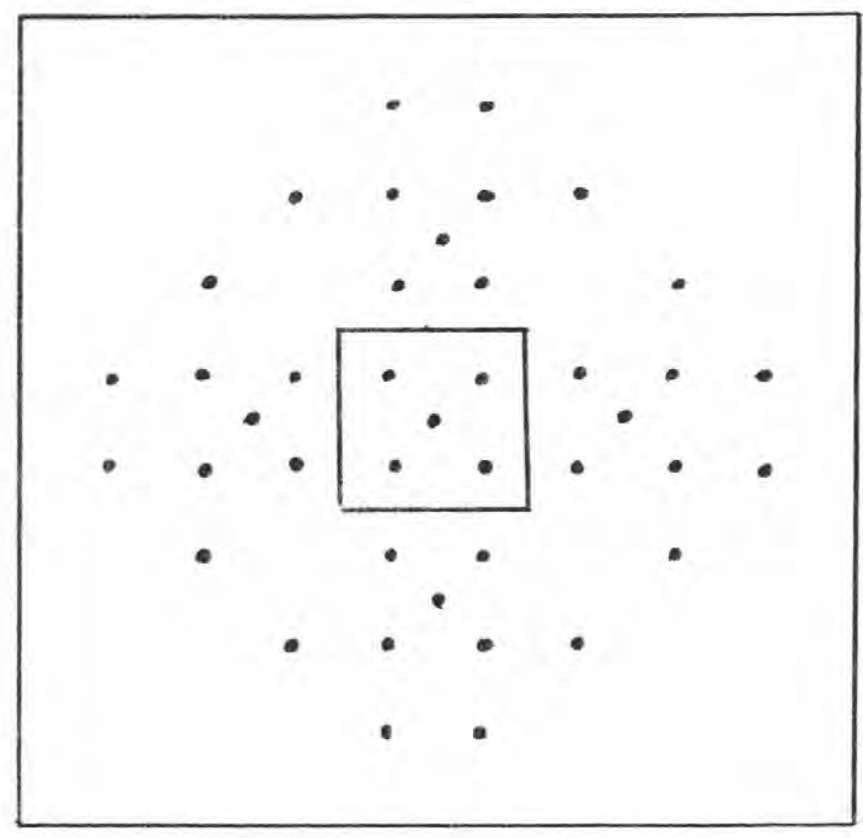

FIGURA 8:

Configuración para aplicar Krigeage a un panel con 5 sondeos interiores. 
Ia solución del sistema de ecuaciones correspondiente condu ce a la siguiente estimación para el panel

$$
z_{p}=z_{k}^{*} \pm 0.23
$$

Es decir, tenemos un error de estimación local para la ley del panel de 0.23 , con 10 cual hemos reducido el error de estimación local en un $56,6 \%$ respecto a los 99 sondeos iniciales $y$ en un $52,8 \%$ respecto a los 219 sondeos.

Indudablemente que podríamos hacer otros tipos de sondeos $y$ así tener una visión mucho más completa en cuanto a las estimaciones locales y globales para el yacimiento.

A modo de resumen podemos presentar el siguiente cuadro, pa ra que los ejecutivos de las empresas mineras decidan el tipo de sondos que desean efectuar, haciendo un estudio costo-precisión

\begin{tabular}{|c|c|c|}
\hline $\begin{array}{l}\text { NUMERO DE } \\
\text { SONDAJES }\end{array}$ & $\begin{array}{l}\text { ERROR } \\
\text { GLOBAL }\end{array}$ & $\begin{array}{l}\text { ERROR } \\
\text { LOCAI }\end{array}$ \\
\hline 99 & 0.085 & 0.53 \\
\hline 219 & 0.047 & 0.48 \\
\hline 495 & 0.034 & 0.23 \\
\hline
\end{tabular}

Como se puede apreciar el errox global es pequeño, luego si la empresa minera solo está interesada en una estimación global del yacimiento bastará con pocos sondeos, pero si desea una estimación lo cal deberá considerar la posibilidad de hacer más sondeos.

Debemos hacer notar que si se decide por un número mayor de sondeos a los 99 ya efectuados, podemos usar los nuevos sondeos para mejorar nuestro semivariograma, y con ello cambian todas las estima- 
ciones que hemos realizado, produciéndose una ganancia mayor aun en la precisión de las estimaciones.

Pero no solo podemos hacer uso del Krigeage en la etapa de sondajes, sino también en plena explotación de la mina, ya que generalmente hay una serie de datos disponibles que podemos usar tales co mo datos obtenidos en pozos de voladuras, en galerías de avance y en paneles ya explotados, etc..

Supongamos ahora que ya hemos explotado en panel $\mathrm{P}_{1} \mathrm{y}$ conoce mos su verdadera ley media, de acuerdo a un informe de la planta. Vea mos como podemos usar esta información para estimar la ley de un panel adyacente $\mathrm{P}_{0}$. Para esta situación debemos usar la siguiente configuración para el Krigeage

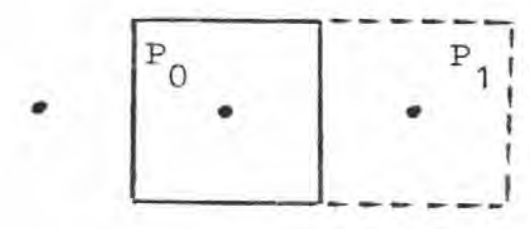

FIGURA 9:

Configuración para la estimación de un panel, usando un panel adyacen te de ley media conocida.

La solución del sistema de ecuaciones nos da el siguiente re sultado

$$
\begin{aligned}
z_{k}^{*}=0.503 z_{1} & +0.080\left(z_{1}+z_{2}+z_{3}+z_{4}\right)+ \\
& +0.012\left(z_{5}+z_{6}+z_{7}+z_{8}\right)+0.129 z_{p}
\end{aligned}
$$

$\sigma_{\mathrm{k}}^{2}=0.05$

$\operatorname{con} \mathrm{Z}=\mathrm{z}_{\mathrm{k}}^{*} \pm 0.45$ 
Veamos ahora el uso práctico de las fórmulas encontradas, aprovechando los 99 sondeos iniciales.

Para la estimación global del yacimiento, tenemos que

$$
\begin{aligned}
& \mathrm{m}=\mathrm{m}_{0}^{*} \pm 0.085 \\
& \mathrm{~m}_{0}^{*}=\frac{\sum_{i=1}^{99} \mathrm{z}_{i}}{99}=2.95 \\
& \mathrm{~m}=2.95 \pm 0.085 \\
& 0 \text { equivalentemente }
\end{aligned}
$$

$$
2.87 \leqslant m \leqslant 3.04
$$

es decir la ley media del yacimiento se encuentra entre 2.87 y 3.04 .

Para la estimación local del bloque achurado de la Figura 2, recordamos que los cálculos nos dieron

$$
\begin{aligned}
& z_{k}^{*}=0.60 z_{1}+0.10\left(z_{2}+z_{3}+z_{4}+z_{5}\right) \\
& z_{p}=z_{k}^{*} \pm 0.53
\end{aligned}
$$

De la Figura 2 tenemos

$$
\begin{gathered}
z_{1}=3, z_{2}=4, z_{3}=2, z_{4}=4 y z_{5}=1 \\
\therefore z_{k}^{*}=0.60(3)+0.10(4+2+4+1) \\
z_{k}^{*}=2.90
\end{gathered}
$$

Luego $\mathrm{z}_{\mathrm{p}}=2.90 \pm 0.53$

Es decir

$$
2.37 \leqslant z_{p} \leqslant 3.43
$$

que nos indica que la ley media del panel achurado está entre 2.37 y 3.43 . 
Puesto que este cálculo podemos hacerlo para cada uno de los 99 paneles, tendremos para cada uno de ellos un intervalo para la ley media del panel y así podríamos dirigir la explotación del yacimiento según los requerimientos de leyes.

Finalmente estimemos la ley del panel doblemente achurado en la Figura 2, supongamos que el panel achurado de la derecha ya ha sido explotado y su ley verdadera es 3.12 usando los últimos calculos tenemos

$z_{k}^{*}=0.503(4)+0.08(2+3+4+3)+0.012(3+4+1+4)+0.129(3.12)$

Es decir $\quad z_{k}^{*}=3.52 \quad y \quad \sigma_{k}^{2}=0.05$

que podemos escribir $3.07 \leqslant z_{p} \leqslant 3.97$

que nos indica que la ley media del panel doblemente achurado está en tre 3.07 y 3.97 .

Si este mismo panel lo hubiésemos estimado antes de explotar el panel achurado la estimación sería con $z_{1}=4, z_{2}=2, x_{3}=3$, $\mathrm{z}_{4}=3 \quad \mathrm{y} \quad \mathrm{z}_{5}=4$.

$z_{k}^{*}=0.60(4)+0.10(2+3+3+4)$

$\mathrm{z}_{\mathrm{k}}^{*}=3.60$ y $\quad \sigma_{\mathrm{k}}^{2}=0.07$

$3.07 \leqslant z_{p} \leqslant 4.13$

Como conclusión podemos afirmar que las técnicas de Geoestadística, nos permite analizar teóricamente (sin explotación) los erro res globales y locales en nuestras estimaciones, como también mejorar estas estimaciones a medida que se va explotando el yacimiento. 
BIBLIOGRAFIA:

ALFARO, M.

ALFARO, M.

MICHEL, DAVID
: Reconocimiento Rígido y Secuencial: Un estudio geoestadístico paso a paso.

: Introducción Práctica a la Geoestadística. De partamento de Minas. Universidad de Chile.

: Geostatistical Ore Reserve Estimation Elsevier Scientific Publishing Company Amsterdam, Osford, New York, 1977.

A. ROYLE, I. CLARK : Geostatistics. MC Graw-Hill, 1980. $y$ otros. 